\title{
A study of the porosity of nuclear graphite using small-angle neutron scattering
}

\author{
Z. Mileeva $^{a, *}$, D.K. Ross ${ }^{a}$, S.M. King ${ }^{b}$ \\ a Materials and Physics Research Centre, Maxwell Building, University of Salford, Manchester M5 4WT, UK \\ ${ }^{\mathrm{b}}$ ISIS Facility, STFC, Rutherford Appleton Laboratory, Didcot, Oxon OX11 OQX, UK
}

\section{A R T I C L E I N F O}

Article history:

Received 24 April 2013

Accepted 12 June 2013

Available online 20 June 2013

\begin{abstract}
A B S T R A C T
Small-angle neutron scattering (SANS) measures porosity in nuclear graphites, including both open pores, caused by escaping decomposition gases, and internal cracks (in coke particles) generated by anisotropic thermal contraction along the c-direction (Mrozowski Cracks). Porosity changes on the length scale observable by SANS must control the development of internal stresses and hence of cracking in AGR graphite due to irradiation (both fast neutron displacements of carbon atoms and radiolytic corrosion by $\mathrm{CO}_{2}$ ). Such cracking may cause premature reactor shutdown. SANS measurements show that porosity is fractal on a length scale between $\sim 0.2$ and $300 \mathrm{~nm}$, presumably due to Mrozowski cracks - because the fractal index of the SANS signal depends only on the porosity of the graphitic filler. We report here two novel uses of the SANS technique as applied to reactor graphite contrast matching with D-toluene (to measure the fraction of the porosity open to the surface) and the temperature dependence of the scattering (to measure pore width changes up to $2000^{\circ} \mathrm{C}$ ). These results provide important new information on AGR graphite porosity and its evolution during irradiation.
\end{abstract}

(C) 2013 Elsevier Ltd. All rights reserved.

\section{Introduction}

Currently about $10 \%$ of the UK electrical generating capacity comes from the Advanced Gas-cooled nuclear Reactors (AGRs) which are scheduled to shut down in the period 2018-2023 although the closure date may be extended, if an acceptable safety case can be established. They are to be replaced with water moderated reactors (PWRs or BWRs). However, progress with the latter is subject to significant delay. Thus, research contributing to the life-extension of the UK AGR fleet could literally keep the UK's lights on. At present, uncertainty in the behavior of the graphite moderators creates a major difficulty in establishing a safe limit to operating life because deterioration of the moderator is one of the key factors limiting the potential lifetimes of $\mathrm{AGRs}^{1}$. After prolonged high intensity irradiation the accumulated fast neutron damage causes microscopic dimensional changes as well as changes in the total porosity [1]. Also, radiolytic corrosion by the carbon dioxide coolant enlarges the pores open to the coolant [2]. These, in turn, change the macroscopic properties of the moderator material making it more fragile and the occurrence of cracking more unpredictable.

A model for the fast neutron damage in graphite was originally developed in the 1970s but this is now believed to be over-simplified because, at high radiation doses, the observed properties differ significantly from those predicted. The traditional view was that irradiation created new inter-

\footnotetext{
* Corresponding author.

E-mail address: z.mileeva@salford.ac.uk (Z. Mileeva).

${ }^{1} \mathrm{http} / /$ www.euronuclear.org/events/enc/enc2012/transactions/ENC2012-transactions-plant-operations.pdf. 0008-6223/\$ - see front matter @ 2013 Elsevier Ltd. All rights reserved. http://dx.doi.org/10.1016/j.carbon.2013.06.030
} 
stitial hexagonal layers which were only weakly coupled to the rest of the graphite structure [1]. However, ab inito calculations demonstrate that the atoms at the edge of such a layer will interact strongly with the layer above or below $[3,4]$. The resulting uncertainty in the behavior of the moderator is considered likely to limit the safe operating life of the reactor.

Currently, there are a number of techniques available to investigate the properties of reactor graphites on both the atomistic and macroscopic scales. However, in order to understand the mechanisms involved, it is necessary to understand a link between the properties on these very different length scales and this link is currently difficult to establish. Small-angle neutron scattering (SANS) measurements can uniquely provide an insight into the graphite structure and its evolution under reactor conditions in the mesoscopic range $(\sim 0.2-300 \mathrm{~nm})$. It should be noted that various kinds of microscopy can be used on these length scales. However, microscopy examines a very limited field of view of this very disordered material whereas SANS records, in one measurement, the average density-density (or porosity-porosity) correlation function over the above length scale - providing a precise description of the average pore width distribution. This technique is easily extended to measure, for instance, the macroscopic variation of this porosity with position in an irradiated graphite component.

\subsection{Small-angle neutron scattering}

Full reviews of the small-angle neutron scattering from carbons can be found elsewhere $[5,6]$. However, it is useful to give the general form of the equation expressing the coherent neutron differential scattering cross-section as a function of the scattering vector, $Q$.

$\frac{d \Sigma}{d \Omega}(Q)=N_{P} V_{P}^{2}\left(\rho_{c}-\rho_{p}\right)^{2} S(Q) F^{2}(Q)+B_{i n c}$

Here $Q=4 \pi \sin (\theta / 2) / \lambda$ where $\theta$ is the scattering angle and $\lambda$ is the neutron wavelength, $\rho_{\mathrm{c}}$ is the neutron scattering length density of the carbon and $\rho_{p}$ is the same quantity for the pore (or the corresponding value when the pore is filled with a liquid), $N_{p}$ is the number of pores/unit volume, $V_{p}$ is the volume of the pore, $S(Q)$ describes the spatial separation of the pores and $F(Q)$ is the form factor which is determined by the pore shape. More formally, these quantities should be averaged over a distribution of pore sizes. Finally, $B_{i n c}$ is a flat $(Q$-independent) background due mainly to incoherent scattering, principally from residual hydrogen.

An important result, known as the Porod Invariant (or Total Scattering), is the integral of the cross-section weighted by $Q^{2}$, the value of which can be related to the volume fraction of the pores, $\varphi$, so long as the cross-section is properly normalized, as it is here [5].

P.I. $=\int_{0}^{\infty} Q^{2} \frac{d \Sigma}{d \Omega}(Q) d Q=2 \pi^{2}\left(\rho_{C}-\rho_{p}\right)^{2} \phi(1-\phi)$

In the present paper, the measured SANS data is presented in terms of $d \Sigma / d \Omega(Q)$, but for simplicity, we refer to this as "Intensity". Values for the Porod Invariant have been determined by integration between the upper and lower limits of the experimental measurements.

\subsection{Previous SANS measurements on reactor graphite}

The SANS technique has been very widely used for the study of activated carbons of all types. This work has been reviewed by Hoinkis [6]. There has been much less work published on denser reactor moderator graphites and this work is reviewed briefly here. The first measurements were by Martin and Henson [7] who measured SANS from reactor graphites before and after irradiation. They found some extra scattering at fairly high $Q$ which they attributed to small clusters of interstitials and vacancies produced by fast neutron irradiation. They also observed that the extra scattering disappeared when samples were annealed at $1100{ }^{\circ} \mathrm{C}$ for one hour. Our present interest is in larger scale porosity and this is seen at considerably lower $Q$ values. Here most of the early measurements were performed by Martin and Caisley [8-10]. These authors measured a series of AGR (Gilsocarbon) graphites, both "as produced" and after neutron irradiation, using the D11 SANS instrument at the ILL in Grenoble [11]. They established that the SANS changed significantly as a result of neutron irradiation [8] and was determined entirely by the coke source used [9]. They also demonstrated how the SANS changed as a result of both thermal and radiolytically-induced oxidation [10]. Data from these experiments, as originally plotted on linear scales of intensity against $Q$, showed a smooth reduction in intensity with increasing $Q$ which was modeled as the sum of two Guinier functions, each of which described the form factor of a spherical pore, averaged over a small range of pore radii (to eliminate terms due to a sharp cutoff at a specific radius) given by:

$\frac{d \Sigma}{d \Omega}(Q) \sim|F(Q)|^{2}=\exp \left(-\frac{1}{3} Q^{2} R_{g}^{2}\right)$

Here, $R_{g}$ is the radius of gyration of the pore (if the pore is truly spherical, $R_{g}{ }^{2}=3 R^{2} / 5$ ). Estimates of the number of pores with radii of gyration near $2.5 \mathrm{~nm}$ and near $10 \mathrm{~nm}$ were derived from a "Guinier Plot", namely a plot of $\ln \left(\frac{d \Sigma}{d \Omega}(Q)\right)$ versus $Q^{2}$ for $Q R_{g}<1$, which would be linear for a single pore size and which would give an intercept of $N_{p}<\mathrm{V}_{p}^{2}>\left(\rho_{\mathrm{c}}-\rho_{p}\right)^{2}$. If there is a distribution of pore radii present, the resultant plot will have a curved form (negative slope decreasing with increasing $Q)$. Thus, the intercepts of the tangents to this curve having gradients corresponding to $R_{g}$ values of $2.5 \mathrm{~nm}$ and $10.0 \mathrm{~nm}$ were taken to be representative of the populations of pores of these sizes. Measurements were made on unirradiated samples and those irradiated with up to $51.3 \times 10^{24} \mathrm{n}$ / $\mathrm{m}^{2}$ of fast neutrons. The results showed a progressive decrease in population of $10.0 \mathrm{~nm}$ pores with a corresponding increase in population of $2.5 \mathrm{~nm}$ pores as a function of irradiation level.

In their second publication [9], Martin and Caisley reported on SANS experiments on 14 different graphite samples. These samples were characterized by the nature of the coke source (petroleum coke, pitch coke or Gilsocarbon) and by a range of other characteristics including the binder material and the grain size of the filler. The very clear conclusion was that the SANS was entirely determined by the nature of the filler. 
This rules out any contribution to the SANS signal from, for instance, the porosity involved in the evolution of gases generated by the pyrolysis of the binder or to cracks in the inter-granular region or at the surface area of the filler, probably because the size of the corresponding pores lie beyond the observed range. In a third paper, they reported on the relative effects of thermal and radiolytic oxidation on the porosity [10].

Finally, Hoinkis et al. [12] compared the effects of oxidative corrosion $\left(\mathrm{CO}_{2}\right.$ at $\left.900^{\circ} \mathrm{C}\right)$ with porosity changes induced by neutron irradiation $\left(5 \times 10^{21} \mathrm{n} / \mathrm{cm}^{2}\right.$ or $\left.\sim 7 \mathrm{dpa}\right)$ in nuclear graphites. Using small angle X-ray scattering, they came to similar conclusions about the changes in the relative populations of large and small pores.

\subsection{Reinterpretation of the SANS data}

Since the publication of Martin and Caisley's data [8-10], the fundamental significance of fractal distributions in Nature has become very widely appreciated [13]. In a "mass fractal", for instance, defined in terms of volume or pore fractals, the density-density correlation function (or the pore-pore correlation function) decreases according to a non-integer power law with an index $D_{m}$ that is less than the Euclidean dimension, $d$. Specifically, it can be shown that $2<D_{m}<3$ in 3dimensions and $0<D_{m}<1$ in 1-dimension. In either case it gives rise to an $S(Q)$ function that varies as $Q^{-D m}$. The term "surface fractal", on the other hand, refers to a fractally rough surface, the area of which increases as the "tile" used to measure it gets smaller. If the surface were smooth (i.e. independent of tile size), the scattering would give rise to a $Q^{-4}$ dependence which is referred to as Porod Scattering. But for a fractal surface, the gradient is reduced below 4, as here higher $Q$ values correspond to a smaller "tile" dimension and hence a larger total area for a fractally rough surface. Formally [14],

$S(Q) \sim Q^{D_{S}-6}$

where $D_{\mathrm{s}}$ is the surface fractal dimension. There are alternative formulations of this equation, arising from rather different assumptions about the interplay between surface and mass fractals $[15,16]$. However, the main value of the fractal picture is that it provides precise power law indices that can be used as a unique characteristic measure of very heterogeneous structures, such as nuclear graphite. We have therefore chosen to use Eq.(4) to describe the fractal properties for short correlation lengths (high Q). We have thus replotted the Martin and Caisley data [8-10] on $\log ($ Intensity) $-\log (Q)$ scales to see whether their samples show the fractal behavior. The plots demonstrate that the SANS from AGR (Gilsocarbon) graphites, does indeed have a non-integer power law form where the exponent varies with fast neutron irradiation (Figs. 1 and 2). This suggests a fractal distribution of porosity that changes in a systematic way as the carbon atoms are displaced by fast neutron irradiation.

Their data for Gilsocarbon graphite type G.M6 are shown in Fig. 1 and give a linear behavior. The scattering from an unirradiated sample clearly exhibits two distinct linear regions with an additional flattening at high $Q$ due to incoherent scattering from hydrogen or other incoherent scattering impuri-

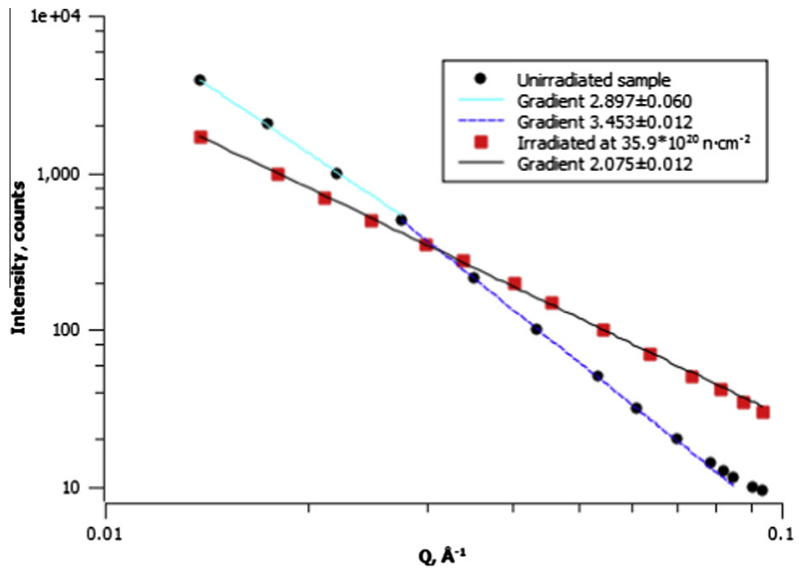

Fig. 1 - SANS from G.M6 graphite: unirradiated and irradiated at $35.9 \times 10^{20} \mathrm{n} \mathrm{cm}^{-2}$ (replotted from Martin and Caisley [8]).

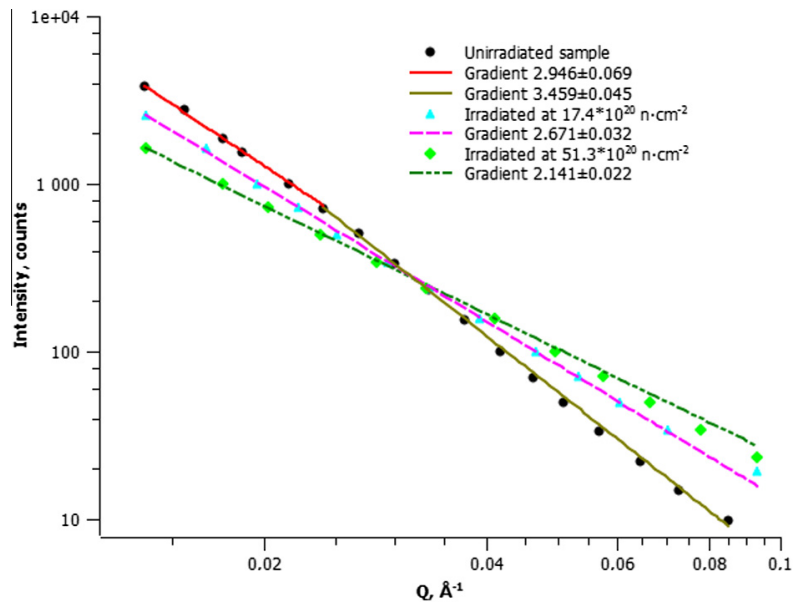

Fig. 2 - SANS from GE1 graphite: effect of irradiation (replotted from [8]).

ties. The gradients are respectively -2.897 at low $Q$ (mass fractal range) and -3.453 at higher $Q$ (surface fractal range). The data for the irradiated sample, remarkably, has changed significantly giving a single linear slope for the full $Q$ range, having a gradient of -2.075 , a mass fractal. Thus, the surface fractal component seems to have been suppressed by the irradiation process (the surface smoothed and reduced in area) while the pore/volume fractal component implies a reduced density of large pores and an increased density of small pores - as in the original interpretation of Martin and Caisley from their derived Guinier radii, using the method described above [8].

It is interesting that the Porod Invariant analysis (Eq.(2) and Ref. [5]) indicates a pore volume/unit volume that increases by $60 \%$ due to irradiation over the measured $Q$ range (between $\sim 67 \AA$ and $\sim 454 \AA\left(2 \pi / Q_{\max }\right.$ and $\left.2 \pi / Q_{\text {min }}\right)$ ). This could mean either that new pores are being formed or alternatively that pore surfaces are being smoothed and larger pores, initially beyond the measured range, are partially filled, hence becoming observable in this $Q$ range. 
The effect of progressive increases in the fast neutron irradiation dose on the power-law exponent is demonstrated in Fig. 2 for GE1 graphite. The initial two-component gradient of the virgin graphite becomes a single line having a decreasing gradient with increasing irradiation. Porod Invariant analysis of the GE1 graphite data also shows a tendency for the volume of small pores to increase by ca. $23 \%$ and $29 \%$ as compared to virgin graphite - for doses of $17.4 \times 10^{20} \mathrm{n} \mathrm{cm}^{-2}$ and $51.3 \times 10^{20} \mathrm{n} \mathrm{cm}^{-2}$ respectively. The original paper includes data on a range of different isotropic graphites which all appear to behave in a similar way.

In another paper [10], these authors reported on the effect on the scattering from electro-graphite blocks - made from both petroleum coke and Gilsocarbon - before and after ther$\mathrm{mal}$ and radiolytic oxidation. For both types of material, thermal oxidation produced an increase in scattering while radiolytic oxidation produced a decrease. In both cases, the effect became more pronounced for the smaller pores (as evidenced by the high $Q$ scattering). Based on the current understanding of the process by which thermal oxidation produces activated carbons, we suggest that an increase in the scattering is due to a much increased population of small pores and hence increased surface area. On the other hand, radiolytic oxidation - the process responsible for mass loss of graphite in reactors due to gamma radiation and $\mathrm{CO}_{2}$ coolant oxidation - enlarges pores in proportion to their size [2] and in the process reduces the population of smaller pores and hence reduces SANS intensity at higher $Q$.

Further, recent measurements on SANS from dense isotropic graphite have been reported by Hoinkis and Allen [17]. These authors measured the scattering from graphite intended for use as the first wall of a fusion reactor - designated POCO AXM-5Q1. This material was binder-free and consisted of nearly perfect graphite grains which were hot pressed using a proprietary method. Measurements were made for a series of burn-offs measured in weight \%. The angular cross section followed a $Q^{-3.1}$ behavior over some 3.5 decades in intensity with a peak at around $0.01 \AA^{-1}$. Oxidation of this graphite to a mass loss of between $0 \%$ and $2.3 \%$ showed a slow $Q$-independent increase in the scattering intensity without any significant change in shape (fractal index and peak position). The power law index lies just within the range of a surface fractal. Alternatively, it would be consistent with a power law form for a pore radial distribution function [15], $F(r) \sim r^{-0.9}$.

\section{Measurements on Gilsocarbon and PGA graphite}

\subsection{Contrast matching}

In the present experiments we have used the SANS technique previously developed for studies of activated carbon [5]. In particular, we have demonstrated that deuterated toluene also provides an excellent contrast matching liquid for graphites, condensing into all pores connected to the surface down to the minimum observable pore dimension $(\sim 0.2 \mathrm{~nm})$. Scattering from all open pores is thus essentially eliminated. The technique has been used to distinguish between open

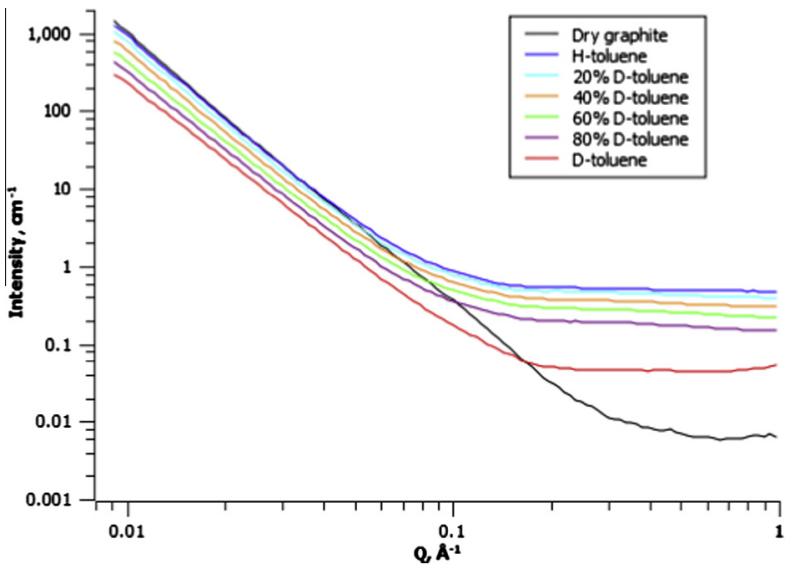

Fig. 3 - Gilsocarbon contrast matching.

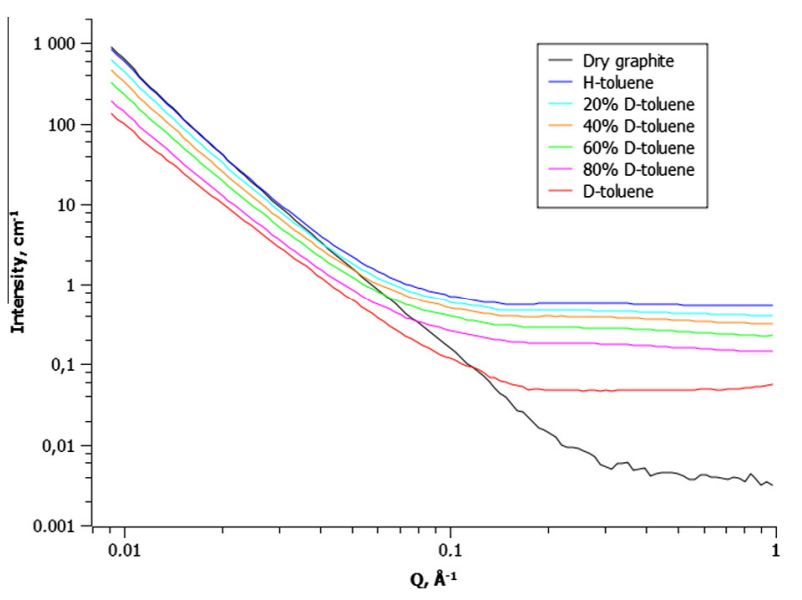

Fig. 4 - PGA graphite contrast matching.

and closed pores within the graphite specimens. These measurements were performed using the LOQ SANS instrument at ISIS [18] covering the $Q$ range from 0.009 to $1.0 \AA^{-1}$. The graphite samples were cut to dimensions of $2.0 \mathrm{~cm} \times$ $2.0 \mathrm{~cm} \times 0.2 \mathrm{~cm}$. Each sample was out-gassed at $200^{\circ} \mathrm{C}$ prior to the measurements - to eliminate as much hydrogenous material as was practicable. Gilsocarbon and PGA (Pile Grade A) graphite samples were studied. Gilsocarbon filler particles have an onion shape which produces rather isotropic properties. PGA graphite is produced from needle shaped coke particles that become aligned along the extrusion direction during manufacture, giving rise to the anisotropic properties of this graphite. Hence, the PGA samples were cut in two ways: parallel and perpendicular to the extrusion direction - to investigate the properties in both. As $Q$ lies in the plane of the sample, we expected the perpendicular cut to give a circularly-symmetric intensity contour plot and the parallel cut to give ellipsoidal contours.

Figs. 3 and 4 demonstrate the contrast match data for Gilsocarbon and PGA graphite respectively. The scattering from the D8-toluene itself is a horizontal line at $\sim 0.1 \mathrm{~cm}^{-1}$ and is omitted from the graphs for clarity. The Porod Invariant analysis of the empty graphite in comparison with that for the same sample saturated with D8-toluene indicates that 


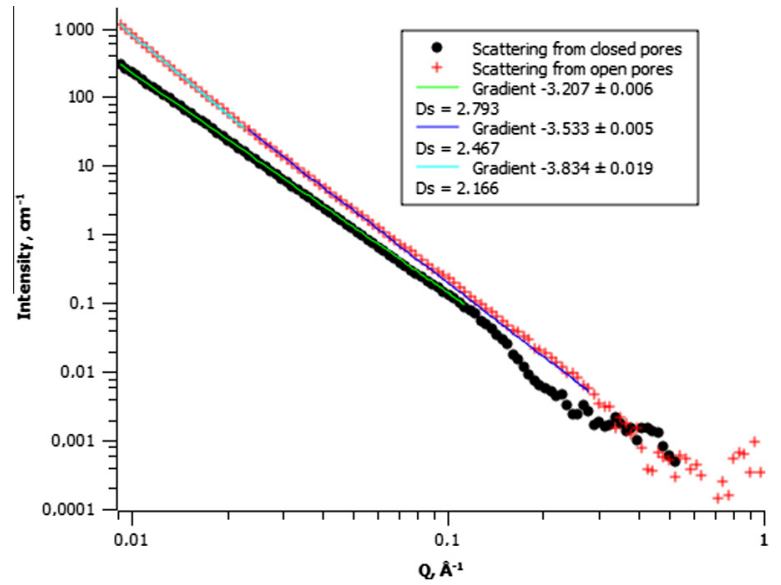

Fig. 5 - Analysis of SANS from open and closed porosity for Gilsocarbon.

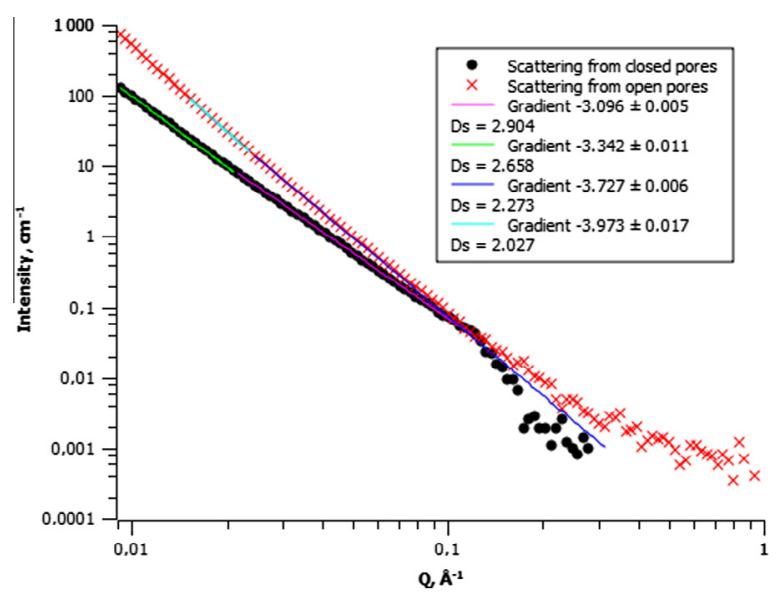

Fig. 6 - Analysis of SANS from open and closed porosity for PGA graphite.

$66 \%$ of porosity in Gilsocarbon and $71 \%$ of that in PGA graphite are accessible to the external surface over the range of length scales measured. Note that the figures show results for different H/D ratios. These data confirm that the best possible matching is for $100 \%$ D8-toluene. The flat background at high $\mathrm{Q}$ clearly increases, as expected, with $\mathrm{H}$ content of the D8/H8-toluene mixtures.

The other significant result from these measurements is that the SANS profiles from the open and closed pores do not coincide with each other, indicating that their pore size distribution as well as their origins are different. Thus, if we subtract the scattering from the samples containing the contrast matching liquid (i.e. corresponding to scattering from closed pores) from the original scattering, we must be left with the scattering from the open pores (this subtraction of intensity depends on there being no interference terms arising from adjacent filled and empty pores). The comparisons of scattering from open and closed pores in each graphite are shown in Figs 5 and 6.

The SANS from the contrast matched samples, i.e. from the closed pores, in Gilsocarbon (Fig. 5) shows a single component power-law with a gradient of -3.207 (surface fractal dimension $D_{s}=2.793$ ), indicating a rather rough surface. PGA closed porosity apparently shows two power laws but data from an extended $Q$ range is needed in order to establish this fully (Fig. 6). The SANS signals from PGA and Gilsocarbon "open porosity" are more complicated and consist of at least two linear regions on a double logarithmic scale. Both imply the presence of smooth surfaces at low $Q$ values and of rougher (fractal) surfaces at higher $Q$ values (Figs. 5 and 6). It is noticeable that there are more large pores in the open pore scattering, as one might expect. We would anticipate the signal from the open pores to increase after the radiolytic corrosion and to indicate whether the corrosion has mainly affected large or small pores. Because erosion is caused by an activated molecular radical species of limited lifetime, the rate of erosion depends on the amount of this radical produced within about a micron range of the pore surface and this strongly enhances the relative importance of small pores [2]. For such pores, as measured here, the erosion effect will be proportional to the volume (width) of the pore so it is not surprising that the SANS signal remains fractal.

\subsection{Temperature dependence}

In the second set of measurements, the samples were mounted in a furnace and heated to $2000^{\circ} \mathrm{C}$ in increments of $200^{\circ} \mathrm{C}$. The resulting SANS signal was measured in situ for each temperature at equilibrium. The identical intensity profiles of the scattering at a given temperature both heating and cooling rules out any observable annealing process in this temperature range. Here we obtain the important result that the SANS intensity decreases in a linear fashion with increasing temperature (Fig. 7) due, presumably, to the filling of cracks normal to the c direction that were formed by thermal contraction during cooling (Mrozowski cracks [19]). Noticeably, although the trend is identical for the two PGA samples (cut parallel and perpendicular to the extrusion direction) the absolute intensities are different - being higher for the sample cut perpendicular due to preferred orientation of the needle coke particles. This orientational aspect of the results will be fully described elsewhere [20]. However, the fractional

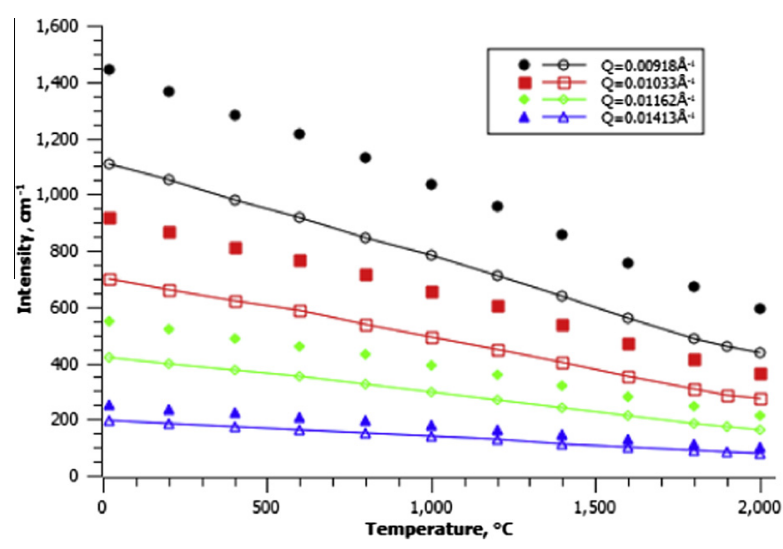

Fig. 7 - The variation with temperature of the scattering intensity from PGA graphite at a set of $Q$ values. Filled and unfilled symbols refer to data from samples cut normal and parallel to the extrusion direction respectively. 


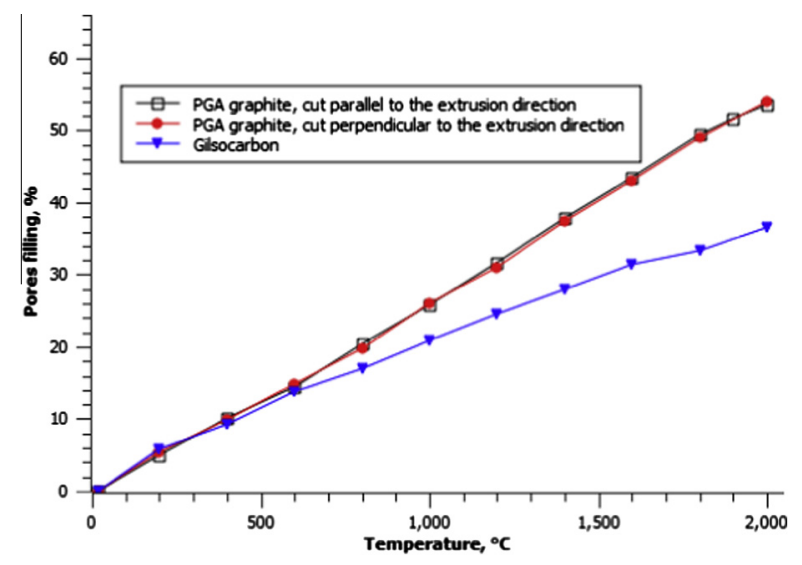

Fig. 8 - Fractional pore closure of PGA and Gilsocarbon graphites.

decrease in the SANS signal for Q-parallel to the extrusion direction relative to that normal is independent of the value of $Q$, suggesting that the porosity distribution is isotropic within a given coke particle. Furthermore, the results suggest that the material would have to be heated to well above $2000^{\circ} \mathrm{C}$ to completely close the pores, suggesting that these pores must have been created due to loss of plasticity in the grist rather than in the binder.

Fig. 8 shows the fractional closure of pores determined from the Porod Invariant values as a function of temperature relative to ambient temperatures in PGA and Gilsocarbon graphites. The pore filling process for the two samples is similar for temperatures up to $600^{\circ} \mathrm{C}$ but starts to deviate with further heating. The pore closure in PGA graphite is essentially linear up to $2000^{\circ} \mathrm{C}$ whereas, in Gilsocarbon, the rate of closure slows down as the temperature increases above $600^{\circ} \mathrm{C}$.

\section{Interpretation of the SANS signal from reactor graphites}

It is apparent that in reactor graphites, the SANS signal arises from the porosity. There are a number of different kinds of porosity present. Some pores in the binder will be interconnected to the surface because they provided an escape route for hydrocarbon gases produced during the carbonization of the binder material. Microscopy also shows cracks around the edges of the graphitic particles [21], and some sets of parallel cracks which are thought to form in the filler particles during cooling from their minimum temperature for plastic deformation. We believe that the SANS signal is determined by these cracks forming in the filler during cooling. The anisotropic thermal contraction in the c direction would be expected to cause the basal planes to pull apart randomly, thus forming cracks parallel to the basal plane which appear with variable widths and separations of the order of 10$100 \mathrm{~nm}$ wide (Mrozowski cracks). Given that the SANS from graphites is determined by the filler particles in use [9] and is described by a single fractal behavior, it is reasonable to assume that the SANS signal is generated by these cracks. Note that the widths of these cracks and their separation appar- ently vary over at least an order of magnitude. Although some planar cracks are visible in the micrographs, these are apparently relatively rare. In fact, the images are dominated by rather disordered structures and the visible cracks have to be rather large to be seen in the SANS [21,22]. It is notable that the fractal index we observe is consistent with a 3-d porosity distribution, not a 1-d distribution. If the cracks were indeed 1-dimensional as observed in the direction normal to the graphite planes, the fractal index would be less than 1 . The larger value observed suggests that most of the domains containing ordered graphitic structure are themselves part of a 3- $d$ structure and that the size of the graphitic particle in the c direction largely determines the total crack width in the particle. The fractal dimension being as for a 3-d arrangement suggests that there are only a few slits in each particle.

Note that the linear decrease in the SANS intensity with increasing temperature is entirely consistent with this model - i.e. the observed porosity was produced by the thermal contraction. This picture is very similar to that described by Hacker et al. [22] who observed that the coefficient of thermal expansion was not influenced by radiolytic weight loss up to quite high weight losses, suggesting that a rigid disordered carbon framework generated the 3-dimensional structure capable of transmitting thermal strains and that the oxidation process probably involved a percolating network of cracks. Given the origin of these cracks, it is remarkable that our contrast matching measurements demonstrate that some $70 \%$ are open to the surface. The distribution of open porosity within the graphite is of great importance in predicting performance when undergoing radiolytic corrosion. An arrangement of macroscopic regions in the graphite block containing open porosity alternating with regions having closed porosity might generate local variation in the macroscopic properties. Thus, given that fast neutron irradiation tends to fill the sealed pores while radiolytic corrosion tends to open out the open pores, the combination of these processes could produce local stress gradients, possibly lead to cracking.

\section{Conclusions}

Previous measurements on reactor graphites have shown a strong SANS signal from graphite over a wide distribution in $\mathrm{Q}$. We have reported here that this scattering from Gilsocarbon and PGA graphites gives a rather good straight line fit to the measured intensity when plotted on a log-log scale, suggesting that the scattering comes from a fractal distribution of pores. We have here extended these earlier measurements using contrast matching to distinguish between filled and empty pores and have, in addition, observed that the SANS decreases linearly with increasing temperature. We would therefore suggest that the SANS signal is dominated by scattering from Mrozowski cracks produced by anisotropic shrinkage of the graphite filler particles during the cooling process. The temperature dependence of the SANS suggests that the pores would be completely closed by around $3000^{\circ} \mathrm{C}$ due to the anisotropic thermal expansion. We can conclude that around $70 \%$ of the porosity is accessible to the external surface, and hence 
to the active species that causes the radiolytic corrosion process, a fact that is highly significant for understanding this process.

\section{Acknowledgements}

The authors are very grateful to Profs. Marsden and Mummery and Dr. Jones of the Manchester Graphite Group for the provision of samples. They are also grateful to the ISIS Facility for the provision of neutron beam time on LOQ Experiment number RB1210270. They also wish to acknowledge support from EPSRC (grant EP/I003223/1) as part of the FunGraph consortium (http://www.nuclear-graphite.org.uk/).

\section{R E F E R E N C E S}

[1] Kelly BT. The physics of graphite. London and New Jersey: Applied Science Publishers; 1981; ISBN 0-85334-960-6, p. 386468.

[2] Minshall PC, Sadler IA, Wickham AJ. IAEA conference, 1995; IAEA-TECDOC-90: p. 181-191.

[3] Latham CD, Heggie MI, Gámez JA, Suárez-Martínez I, Ewels CP, Briddon PR. The di-interstitial in graphite. J Phys: Condens Matter 2008;20:395220.

[4] Heggie MI, Suarez-Martinez I, Davidson C, Haffenden G. Buckle, ruck and tuck: a proposed new model for the response of graphite to neutron irradiation. J Nucl Mat 2011;413(3):150-5.

[5] Mileeva Z, Ross DK, Wilkinson D, King SM, Ryan T and Sharrock $\mathrm{H}$. The use of small angle neutron scattering with contrast matching and variable adsorbate partial pressures in the study of porosity in activated carbons. Carbon 2012;50:5062-5075.

[6] Hoinkis E. Small angle scattering of neutrons and X-rays from carbons and graphites. In: Thrower PA, Chemistry and physics of carbon, vol. 25. 1997. p. 71.

[7] Martin DG, Henson RW. The scattering of long wavelength neutrons by defects in neutron-irradiated graphite. Phil Mag 1964;9:659-72.
[8] Martin DG, Caisley J. Some studies of the effect of irradiation on the neutron small angle scattering from graphite. J Carbon 1977;15:251-5.

[9] Martin DG, Caisley J. The inference of the coke source of nuclear graphites from neutron small-angle scattering measurements. J Nucl Mater 1977;67:318-9.

[10] Martin DG, Caisley J. Some studies on the effect of thermal and radiolytic oxidation on the neutron small angle scattering from graphite. Carbon 1978;16:199-203.

[11] Ibel K, Schmatz W, Springer T. Theory of a velocity focusing instrument for neutron small angle scattering. Atomkernenergie 1971;17:15-8.

[12] Hoinkis E, Eatherly WP, Krautwasser P, Robins E. Corrosionand irradiation-induced porosity changes of a nuclear graphitic material. J Nucl Mater 1986;141-143:87-95.

[13] Mandelbrot B. The fractal geometry of nature. New York: W. H. Freeman and Co.; 1982.

[14] Bale HD, Schmidt PW. Small-angle X-ray scattering investigation of sub-microscopic porosity with fractal properties. Phys Rev Lett 1984;53:596-9.

[15] Winter RA, Gabke A, Czeslik C, Pfeifer P. Power law fluctuations in phase-separated liquid membranes. Phys Rev E 1999;60:7354-9.

[16] Pfeiffer, P. Small angle scattering laws for intermediates between surface, mass and pore fractals in "Multiple scattering of waves in random media and random rough surfaces". The Pennsylvania State University: 1985.

[17] Hoinkis E, Allen AJ. A study of porosity and interfacial area in original and oxidized POCO graphite AXM-5Q1 using small angle neutron scattering. Carbon 1991;29:81-91.

[18] Heenan RK, Penfold J, King SM. SANS at Pulsed Neutron Sources: Present and Future Prospects. J Appl Crystallogr 1997;30:1140-7.

[19] Mrozowski S. In: Proceedings of the first and second conferences on Carbon, New York, NY, USA: University of Buffalo; 1956.

[20] Mileeva Z, Ross DK, King S, Jones A, Marsden B, Mummery P. To be published.

[21] Wen K, Marrow J, Marsden B. Microcracks in nuclear graphite and highly oriented pyrolytic graphite (HOPG). J Nucl Mater 2008;381:199-203.

[22] Hacker PJ, Neighbour GB, McEnaney B. The coefficient of thermal expansion of nuclear graphite with increasing thermal oxidation. J Phys D: Appl Phys 2000;33:991-8. 